\title{
Diatoms (Bacillariophyta) associated with lichens from Ulu Peninsula (James Ross Island, NE Antarctic Peninsula)
}

\author{
Barbora Chattová* \\ Department of Botany \& Zoology, Faculty of Science, Masaryk University, Kotlářrská 2, \\ 61137 Brno, Czech Republic
}

\begin{abstract}
Since 2000, the entire Antarctic diatom flora is being revised using a more fine-grained taxonomy based on a better analysis and interpretation of the morphological and molecular observations. Despite the increased diatom research and efforts, the diversity and ecology of diatoms of lichen inhabiting flora of James Ross Island weren't studied yet. To reveal the actual diatom diversity, samples were collected during February and March 2018 from lichens on the Ulu Peninsula, James Ross Island, a 2,450 km² large island, situated in the north-western part of the Weddell Sea, close to the northern tip of the Antarctic Peninsula. The analysis of 29 lichen samples revealed the presence of 56 diatom taxa belonging to 17 genera. The most abundant species were Luticola muticopsis, Hantzschia amphioxys f. muelleri, Pinnularia borealis var. scalaris, Luticola aff. pusilla and Achnanthes muelleri. Biogeographically, the lichen-inhabiting diatom flora of the Ulu Peninsula is composed of cosmopolitan, Antarctic and endemic elements. The present study is the first focusing on the diversity of lichen-inhabiting diatom communities on James Ross Island, revealing the presence of a rather species rich diatom flora.
\end{abstract}

Key words: Antarctica, diatoms, diversity, James Ross Island, lichens, Ulu Peninsula

DOI: $10.5817 / \mathrm{CPR} 2018-2-12$

\section{Introduction}

Diatoms are eukaryotic unicellular microorganisms, characterised by its unique, silica-impregnated cell wall known as a frustule and are one of the most abundant groups of micro-algae present in the Antarctic Region (Jones 1996, Van de Vijver et Beyens 1999a). Although the majority of diatom species are bound to aquatic habi- tats, a large number of diatom taxa are able to survive in non-submerged or even dry habitats such as dry mosses, humid rocks, soils and lichens (Van de Vijver et Beyens 1999b, Zidarova et al. 2010). Lichen communities represent an important part of the Antarctic vegetation and belong to principal components of the terrestrial ecosystem

Received September 5, 2018, accepted December 11, 2018.

"Corresponding author: B. Chattová < barbora.chattova@gmail.com>

Acknowledgements: The author would like to thank the scientific infrastructure of the J.G. Mendel Czech Antarctic Station and its crew for their support (project CzechPolar2, LM2015078). Sincere thanks go to Dr. Filip Hrbáček for his valuable comments on the manuscript. 
of James Ross Island (Bohuslavová et al. 2012). Diatoms have shown the ability to colonize lichens via aerial dispersal (Bertrand et al. 2016), but the diatom diversity has been rarely studied and papers focusing on lichen inhabiting diatom flora are extremely scarce. Dodd et Stoermer (1962) were the first to investigate diatom flora associated to a lichen Collema sp. in Boone Country, Iowa. In 2004 Lakatosh et al. conducted a research on diatoms living inside the thallus of adnate corticolous lichens in neotropical lowland rain forests and finally in 2016 Bertrand et al. published a prelimi-

\section{Material and Methods}

\section{Study site}

James Ross Island (64ำ'S, $\left.57^{\circ} 45^{\prime} \mathrm{W}\right)$, a large island with a total surface area of $2450 \mathrm{~km}^{2}$, is located in the northwestern part of the Weddell Sea, close to the northern tip of the Antarctic Peninsula (Fig. 1). The mean annual temperature is around $-7.0^{\circ} \mathrm{C}$ (Ambrožová et Láska 2016). More than $75 \%$ of its area is covered with a glacier (Rabassa et al. 1982). The largest icefree area, Ulu Peninsula $\left(310 \mathrm{~km}^{2}\right)$ is locat-

\section{Fieldwork and sample preparation}

29 lichen samples were collected during a field campaign in February and March 2018. The samples were collected in PVC bottles. Each sample was geographically localized using GPS and was accompanied by a detailed site description. Table 1 lists all samples together with their geographic co-ordinates. Photographs of selected sampling sites with the lichens are presented at Fig. 2. Light microscope observations directly at the Mendel station were conducted using an Olympus CX31 microscope. All samples were scanned under a microscope before fixing with 3\% formaldehyde and the dominating cyanobacterial and al- nary study on diatoms associated with five lichen genera. The most recent ecological surveys on James Ross Island were dealing with the freshwater, semi-aquatic, mossinhabiting and soil diatom communities of the Ulu Peninsula (Kopalová et al. 2012, Kopalová et al. 2013, Kopalová et al. 2014, Chattová et al. 2016). Despite the increased diatom research and efforts, the diversity and ecology of lichen inhabiting flora of James Ross Island weren't studied yet. One of the main objectives of this study is to bring new information about diatoms associated with lichens from Ulu Peninsula.

ed in the northern part of the island (Kavan et al. 2017) and forms the largest ice-free area in the Antarctic Peninsula region (Hrbáček et al. 2017). Vascular plants are absent, the vegetation is limited to a bryophyte and lichen tundra (Barták et al. 2016). The human presence on the island is limited to its northern part, where the Johann Gregor Mendel Czech Antarctic Station is located since 2006 .

gal flora was listed. The fixed material was taken into Czech Republic, where the permanent diatom slides were prepared for further analysis following the method described in Van der Werff (1955): small parts of the samples were cleaned by $37 \%$ $\mathrm{H}_{2} \mathrm{O}_{2}$ and heated to $80^{\circ} \mathrm{C}$ for about $1 \mathrm{~h}$.

The reaction was completed by addition of $\mathrm{KMnO}_{4}$. Following digestion and centrifugation (five times $10 \mathrm{~min}$. at $3500 \times \mathrm{g}$ ), cleaned material was diluted with distilled water to avoid excessive concentrations of diatom valves on the slides. Cleaned diatom valves were mounted in Naphrax ${ }^{\circledR}$. 

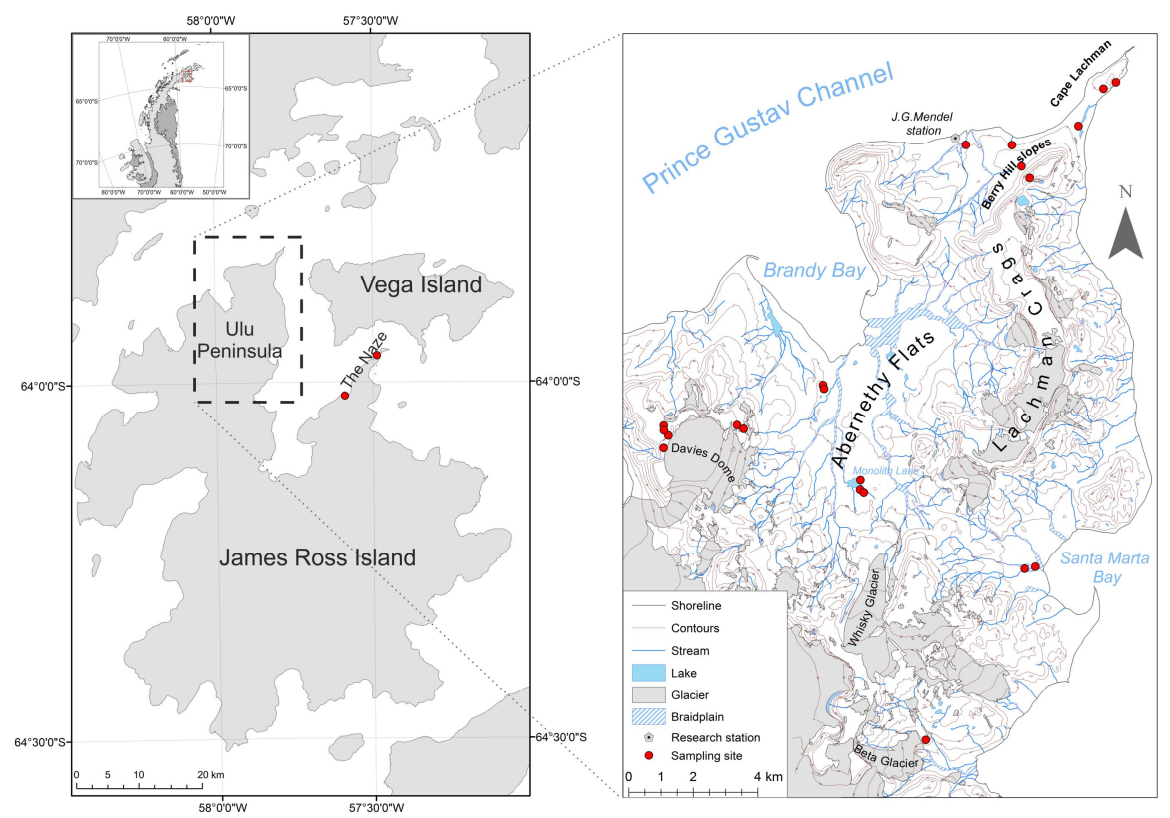

Fig. 1. Map of James Ross Island ([1] - Antarctic Digital Database 2018) and a modified map of James Ross Island-Northern part ([2]- Czech Geological Survey 2009) with indication of sampling sites. Credits: Filip Hrbáček.

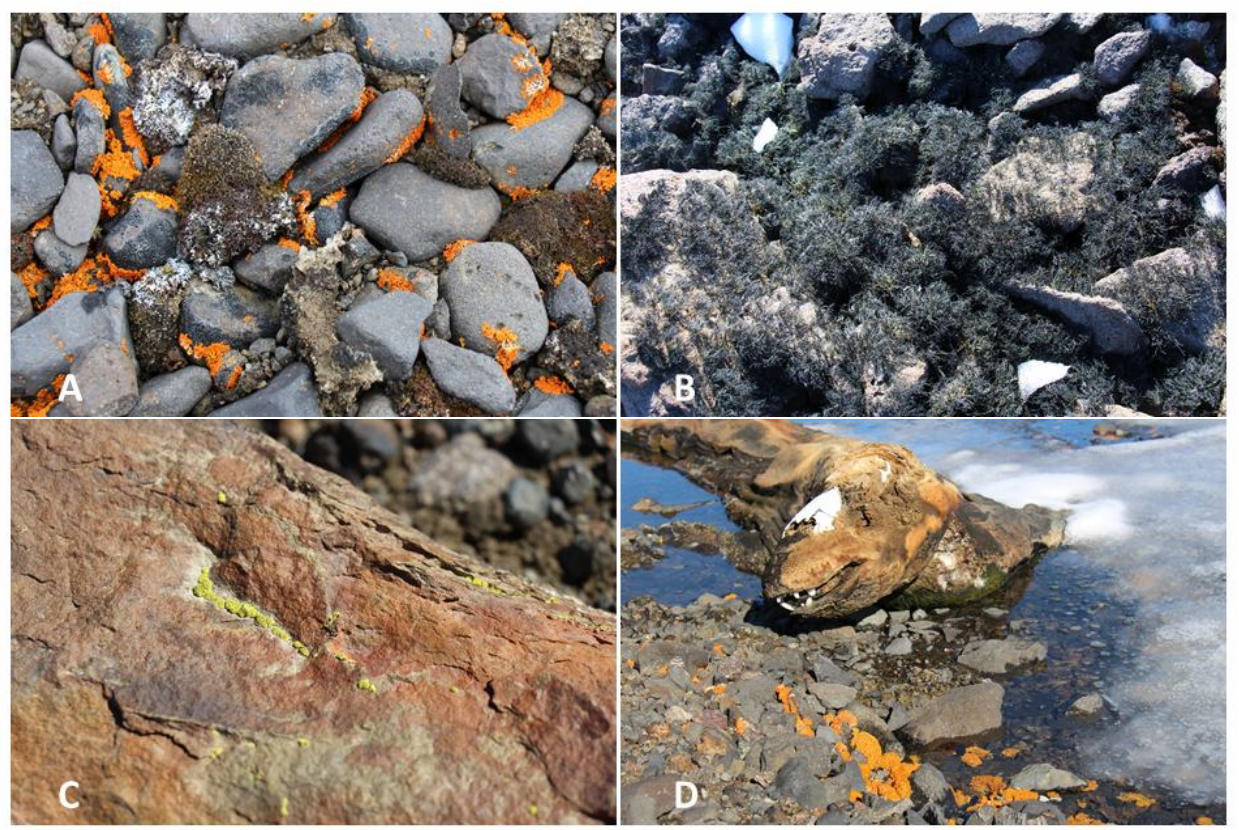

Fig. 2. General view on sampling site No. 26 Xanthoria sp. (A), sampling site No. 9 with Usnea antarctica (B), sampling site No. 6 with Candelariella aff. flava (C), sampling site No. 13 with Xanthoria sp. (D). 


\begin{tabular}{|c|c|c|c|c|c|}
\hline & Locality & Date & GPS & $\begin{array}{l}\text { Eleva- } \\
\text { tion }\end{array}$ & Type of lichen \\
\hline Lich1* & Abernethy Flats & 3.2 .2018 & $\begin{array}{l}63^{\circ} 52^{\prime} 15^{\prime \prime} \mathrm{S} \\
57^{\circ} 58^{\prime} 26^{\prime \prime} \mathrm{W}\end{array}$ & 69 & Usnea antarctica \\
\hline Lich2* & Abernethy Flats & 3.2 .2018 & $\begin{array}{l}63^{\circ} 52^{\prime} 16^{\prime \prime} \mathrm{S} \\
57^{\circ} 58^{\prime} 27^{\prime \prime} \mathrm{W}\end{array}$ & 69 & Usnea antarctica \\
\hline Lich3 & Abernethy Flats & 3.2 .2018 & $\begin{array}{l}63^{\circ} 53^{\prime} 59^{\prime \prime} \mathrm{S} \\
57^{\circ} 57^{\prime} 16^{\prime \prime} \mathrm{W}\end{array}$ & 74 & Xanthoria sp. \\
\hline Lich4 & Monolith Lake & 3.2 .2018 & $\begin{array}{l}63^{\circ} 53^{\prime} 58^{\prime \prime} \mathrm{S} \\
57^{\circ} 57^{\prime} 16^{\prime \prime} \mathrm{W}\end{array}$ & 71 & Xanthoria sp. \\
\hline $\operatorname{Lich} 5^{*}$ & $\begin{array}{l}\text { Under Davies Dome } \\
\text { Glacier }\end{array}$ & 5.2 .2018 & $\begin{array}{l}63^{\circ} 52^{\prime} 52^{\prime \prime ~ S} \\
58^{\circ} 011^{\prime} 54^{\prime \prime} \mathrm{W}\end{array}$ & 397 & Usnea antarctica \\
\hline Lich6* & $\begin{array}{l}\text { Under Davies Dome } \\
\text { Glacier }\end{array}$ & 5.2 .2018 & $\begin{array}{l}63^{\circ} 52^{\prime} 52^{\prime \prime} \mathrm{S} \\
58^{\circ} 01^{\prime} 44^{\prime \prime} \mathrm{W}\end{array}$ & 397 & $\begin{array}{l}\text { Candelariella aff. } \\
\text { flava }\end{array}$ \\
\hline Lich7 & Davies Dome Mesa & 5.2 .2018 & $\begin{array}{r}63^{\circ} 53^{\prime} 20^{\prime \prime} \mathrm{S} \\
58^{\circ} 04^{\prime} 33^{\prime \prime} \mathrm{W}\end{array}$ & 426 & Usnea antarctica \\
\hline Lich8* & $\begin{array}{l}\text { Near OTC Davies Dome } \\
\text { Mesa }\end{array}$ & 5.2 .2018 & $\begin{array}{l}63^{\circ} 53^{\prime} 02^{\prime \prime} \mathrm{S} \\
58^{\circ} 04^{\prime} 17^{\prime \prime} \mathrm{W}\end{array}$ & 512 & $\begin{array}{l}\text { Candelariella aff. } \\
\text { flava }\end{array}$ \\
\hline Lich9* & $\begin{array}{l}\text { Near Meteo station Davies } \\
\text { Dome }\end{array}$ & 5.2 .2018 & $\begin{array}{l}63^{\circ} 52^{\prime} 49^{\prime \prime} \mathrm{S} \\
58^{\circ} 04^{\prime} 37^{\prime \prime} \mathrm{W}\end{array}$ & 496 & Usnea antarctica \\
\hline Lich10* & Davies Dome Glacier & 5.2 .2018 & $\begin{array}{l}63^{\circ} 52^{\prime} 48^{\prime \prime} \mathrm{S} \\
58^{\circ} 04^{\prime} 40^{\prime \prime} \mathrm{W}\end{array}$ & 534 & $\begin{array}{l}\text { Candelariella aff. } \\
\text { flava }\end{array}$ \\
\hline Lich11* & $\begin{array}{l}\text { Monolith Lake, near dead } \\
\text { seal T3 }\end{array}$ & 9.2 .2018 & $\begin{array}{l}63^{\circ} 53^{\prime} 49^{\prime \prime} \mathrm{S} \\
57^{\circ} 56^{\prime} 58^{\prime \prime} \mathrm{W}\end{array}$ & 86 & $\begin{array}{l}\text { Candelariella aff. } \\
\text { flava }\end{array}$ \\
\hline Lich12 & $\begin{array}{l}\text { Monolith Lake, near dead } \\
\text { seal T3 }\end{array}$ & 9.2 .2018 & $\begin{array}{l}63^{\circ} 53^{\prime} 49^{\prime \prime} \mathrm{S} \\
57^{\circ} 56^{\prime} 58^{\prime \prime} \mathrm{W}\end{array}$ & 86 & Xanthoria sp. \\
\hline Lich13 & $\begin{array}{l}\text { Monolith Lake, next to } \\
\text { dead seal T3 }\end{array}$ & 9.2 .2018 & $\begin{array}{l}63^{\circ} 53^{\prime} 53^{\prime \prime} \mathrm{S} \\
57^{\circ} 56^{\prime} 58^{\prime \prime} \mathrm{W}\end{array}$ & 81 & Xanthoria sp. \\
\hline Lich14 & St. Martha Cove & 10.2 .2018 & $\begin{array}{l}63^{\circ} 55^{\prime} 28^{\prime \prime} \mathrm{S} \\
57^{\circ} 50^{\prime} 19^{\prime \prime} \mathrm{W}\end{array}$ & 73 & Xanthoria sp. \\
\hline Lich15* & St. Martha Cove & 10.2 .2018 & $\begin{array}{l}63^{\circ} 55^{\prime} 28^{\prime \prime} \mathrm{S} \\
57^{\circ} 50^{\prime} 53^{\prime \prime} \mathrm{W}\end{array}$ & 62 & Usnea antarctica \\
\hline Lich16 & Cape Lachman & 15.2.2018 & $\begin{array}{l}63^{\circ} 47^{\prime} 02^{\prime \prime} \mathrm{S} \\
57^{\circ} 47^{\prime} 02^{\prime \prime} \mathrm{W}\end{array}$ & 85 & Xanthoria sp. \\
\hline Lich17* & Top of Berry Hill & 16.2 .2018 & $\begin{array}{l}63^{\circ} 48^{\prime} 48^{\prime \prime} \mathrm{S} \\
57^{\circ} 50^{\prime} 43^{\prime \prime} \mathrm{W}\end{array}$ & 364 & Usnea antarctica \\
\hline Lich18* & Cape Lachman & 15.2.2018 & $\begin{array}{l}63^{\circ} 47^{\prime} 02^{\prime \prime} \mathrm{S} \\
57^{\circ} 47^{\prime} 02^{\prime \prime} \mathrm{W}\end{array}$ & 85 & Xanthoria sp. \\
\hline $\operatorname{Lich} 20^{*}$ & Cape Lachman & 15.2.2018 & $\begin{array}{l}63^{\circ} 47^{\prime} 12^{\prime \prime} \mathrm{S} \\
57^{\circ} 47^{\prime} 32^{\prime \prime} \mathrm{W}\end{array}$ & 122 & Usnea antarctica \\
\hline $\operatorname{Lich} 21^{*}$ & Cape Lachman & 15.2.2018 & $\begin{array}{l}63^{\circ} 47^{\prime} 35^{\prime \prime} \mathrm{S} \\
57^{\circ} 47^{\prime} 02^{\prime \prime} \mathrm{W}\end{array}$ & 105 & Usnea antarctica \\
\hline $\operatorname{Lich} 22 *$ & Cape Lachman & 15.2.2018 & $\begin{array}{r}63^{\circ} 47^{\prime} 22^{\prime \prime} \mathrm{S} \\
57^{\circ} 47^{\prime} 72^{\prime \prime} \mathrm{W}\end{array}$ & 53 & Xanthoria sp. \\
\hline Lich23 & The Naze & 27.2.2018 & $\begin{array}{r}63^{\circ} 55^{\prime} 50^{\prime \prime} \mathrm{S} \\
57^{\circ} 30^{\prime} 55^{\prime \prime} \mathrm{W}\end{array}$ & 65 & Xanthoria sp. \\
\hline $\operatorname{Lich} 24$ & Interlagos Lachman & 11.3 .2018 & $\begin{array}{l}63^{\circ} 47^{\prime} 56^{\prime \prime ~ S} \\
57^{\circ} 48^{\prime} 39^{\prime \prime} \mathrm{W}\end{array}$ & 14 & Xanthoria sp. \\
\hline $\operatorname{Lich} 25$ & Berry Hill Slopes & 11.3.2018 & $\begin{array}{l}63^{\circ} 48^{\prime} 17^{\prime \prime} \mathrm{S} \\
57^{\circ} 50^{\prime} 97^{\prime \prime} \mathrm{W}\end{array}$ & 46 & Xanthoria sp. \\
\hline Lich26 & Under Terrapin Hill & 27.2.2018 & $\begin{array}{l}63^{\circ} 58^{\prime} 41^{\prime \prime} \mathrm{S} \\
57^{\circ} 35^{\prime} 32^{\prime \prime} \mathrm{W}\end{array}$ & 4 & Xanthoria sp. \\
\hline $\operatorname{Lich} 27 *$ & Near Beta Glacier & 26.2.2018 & $\begin{array}{r}63^{\circ} 57^{\prime} 58^{\prime \prime} \mathrm{S} \\
57^{\circ} 54^{\prime} 58^{\prime \prime} \mathrm{W}\end{array}$ & 199 & Usnea antarctica \\
\hline
\end{tabular}




\begin{tabular}{|c|c|c|c|c|}
\hline Lich28 & Berry Hill Slopes & 7.3.2018 & $\begin{array}{c}63^{\circ} 48^{\prime} 25^{\prime \prime} \mathrm{S} \\
57^{\circ} 50^{\prime} 33^{\prime \prime} \mathrm{W}\end{array}$ & 146 Xanthoria sp. \\
\hline Lich29 & Berry Hill Slopes & 7.3.2018 & $\begin{array}{c}63^{\circ} 48^{\prime} 24^{\prime \prime} \mathrm{S} \\
57^{\circ} 50^{\prime} 32^{\prime \prime} \mathrm{W}\end{array}$ & 143 Xanthoria $\mathrm{sp}$ \\
\hline Lich30 & $\begin{array}{l}\text { Near JGM station and } \\
\text { Bohemian stream }\end{array}$ & 9.3 .2018 & $\begin{array}{c}63^{\circ} 48^{\prime} 13^{\prime \prime} \mathrm{S} \\
57^{\circ} 52^{\prime} 57^{\prime \prime} \mathrm{W}\end{array}$ & 31 Xanthoria $\mathrm{sp}$ \\
\hline
\end{tabular}

Table 1. List of samples collected on JRI, samples without diatoms are marked with an asterisk*.

In each sample, 200 diatom valves were identified, if possible and enumerated on random transects at $1000 \times$ magnification using an Olympus BX50 microscope (Japan), equipped with Differential Interference Contrast (Nomarski) optics. The diatom taxa were identified as much as possible up to species level or variety. When the taxonomic status of a taxon was uncer-

\section{Results}

During this study, 29 samples from three different lichen species - Usnea antarctica Du Rietz, Xanthoria sp. and Candelariella aff. flava (C.W. Dodge \& Baker) Castello $\&$ Nimis have been analysed, resulting in the observation of a total number of 56 diatom taxa, belonging to 17 genera. Fifteen samples contained (almost) no diatoms, even after counting an entire slide. Subsequently, these samples have been removed from further analysis. Species richness per sample ranged from 7 to 23 with an average number of taxa per sample of 15 . The highest species richness was recorded in sample Lich 25 (23 taxa), a wet Xanthoria sample collected on the north-facing slopes of Berry Hill. This area is supplied by melt water from annual snow deposition and frozen ground. The dominant species were Luticola muticopsis (Van Heurck) D. G. Mann with more than $13 \%$ of all counted valves, followed by Hantzschia amphioxys (Ehrenberg) Grunow f. muelleri Ts. Kobay. (12\%), Pinnularia borealis var. scalaris (Ehrenberg) Rabenhorst (10\%), Luticola aff. pusilla Van de Vijver, Kopalová, Zidarova \& Levkov (9\%) and Achnan- tain, abbreviations 'cf.' (confer: probably belongs to the species identified), 'aff.' (affinis: it bears some similarity to this taxon, but it is not conspecific), or 'sp.' (species of genera given) were used. For identification of Antarctic species mainly the following publications were consulted: Zidarova et al. 2010, 2016, Van de Vijver et al. 2010, 2011, 2014).

thes muelleri Carlson ( $8 \%$ ). Table 2 provides an alphabetical list of all observed species together with their biogeographical distribution. For selected species see Fig. 3. The genera Luticola (fourteen taxa), Humidophila (seven taxa), Nitzschia (six taxa) Hantzschia (five taxa) and Pinnularia (four taxa) were the most species rich genera. Other important genera include Achnanthes, Muelleria and Stauroneis (three taxa). Table 3 lists all genera arranged according to their species number. The majority of diatom taxa were identified from lichens of the genus Xanthoria. Only two single valves of Hanzschia amhioxys and Pinnularia borealis var. scalaris were observed on the thallus of Usnea antarctica. No diatoms were found on Candelariella aff. flava, most likely because the lichen was growing on a dry rock in the most recently deglaciated area of Davies Dome Mesa. Diatoms in the samples were frequently accompanied by various species of genera Leptolyngbya, Oscillaltoria, Cyanothece, Klebsormidium and Nostoc. From a biogeographical point of view, $26 \%$ of the taxa have a typical cosmopolitan distribution, 
with another $5 \%$ species having a restricted distribution to Southern Hemisphere. More than a half of the species $(55 \%)$ are confined to the Maritime Antarctic Region, with additional $14 \%$ showing a restricted distribution to the Antarctic Continent.

\begin{tabular}{|c|c|c|c|}
\hline Species & $\begin{array}{c}\text { Xanthoria } \\
\text { sp. }\end{array}$ & $\begin{array}{c}\text { Usnea } \\
\text { antarctica }\end{array}$ & $\begin{array}{l}\text { Distri- } \\
\text { bution }\end{array}$ \\
\hline Achnanthes coarctata (Brébisson ex W.Smith) Grunow & $\mathrm{x}$ & & $\mathrm{C}$ \\
\hline $\begin{array}{l}\text { Achnanthes muelleri Carlson } \\
\text { Achnanthes taylorensis D.E.Kellogg, Stuiver, T.B.Kellog }\end{array}$ & $\mathrm{x}$ & & $\mathrm{SH}$ \\
\hline \& G.H.D.Denton & $\mathrm{x}$ & & $\mathrm{MA} / \mathrm{CA}$ \\
\hline Brachysira minor (Krasske) Lange-Bertalot & $\mathrm{x}$ & & $\mathrm{C}$ \\
\hline $\begin{array}{l}\text { Caloneis australis Zidarova, Kopalová \& Van de Vijver } \\
\text { Denticula jamesrossensis Van de Vijver, Kopalová, Ector }\end{array}$ & $\mathrm{x}$ & & MA \\
\hline \& Kociolek & $\mathrm{x}$ & & MA \\
\hline Fragilaria cf. parva Tuji \& Williams & $\mathrm{x}$ & & $\mathrm{C}$ \\
\hline Halamphora oligotraphenta Lange-Bertalot \& Levkov & $\mathrm{x}$ & & $\mathrm{C}$ \\
\hline Halamphora sp. (Cleve) Levkov & $\mathrm{x}$ & & $\mathrm{U}$ \\
\hline $\begin{array}{l}\text { Hantzschia abundans Lange-Bertalot } \\
\text { Hantzschia amphioxys (Ehrenberg) Grunow } f \text {. muelleri }\end{array}$ & $\mathrm{x}$ & & C \\
\hline Ts. Kobay. & $\mathrm{x}$ & $\mathrm{x}$ & MA/CA \\
\hline Hantzschia cf. acuticapitata Zidarova \& Van de Vijver & $\mathrm{x}$ & & MA \\
\hline Hantzschia hyperaustralis Van de Vijver \& Zidarova & $\mathrm{x}$ & & $\mathrm{MA} / \mathrm{CA}$ \\
\hline $\begin{array}{l}\text { Hantzschia incognita Zidarova \& Van de Vijver } \\
\text { Humidophila australis (Van de Vijver \& Sabbe) R.L. }\end{array}$ & $\mathrm{x}$ & & MA \\
\hline Lowe et al. & $\mathrm{x}$ & & $\mathrm{MA} / \mathrm{CA}$ \\
\hline $\begin{array}{l}\text { Humidophila contenta group (Grunow) Lowe et al. } \\
\text { Humidophila inconspicua (Kopalová \& Van de Vijver) }\end{array}$ & $\mathrm{x}$ & & $\mathrm{C}$ \\
\hline Lowe et al. & $\mathrm{x}$ & & MA \\
\hline Humidophila aff. ingeae (Van de Vijver) Lowe et al. & $\mathrm{x}$ & & SH \\
\hline Humidophila keiliorum Kopalová & $\mathrm{x}$ & & MA \\
\hline $\begin{array}{l}\text { Humidophila sceppacuersiae Kopalová } \\
\text { Humidophila vojtajarosikii Kopalová, Zidarova \& Van de }\end{array}$ & $\mathrm{x}$ & & MA \\
\hline $\begin{array}{l}\text { Vijver } \\
\text { Chamaepinnularia australomediocris (Lange-Bertalot }\end{array}$ & $\mathrm{x}$ & & MA \\
\hline $\begin{array}{l}\text { \&Smidt) Van de Vijver } \\
\text { Luticola amoena Van de Vijver, Kopalová, Zidarova \& }\end{array}$ & $\mathrm{x}$ & & $\mathrm{MA} / \mathrm{SA}$ \\
\hline Levkov & $\mathrm{x}$ & & MA \\
\hline $\begin{array}{l}\text { Luticola australomutica Van de Vijver } \\
\text { Luticola austroatlantica } \text { Van de Vijver, Kopalova }\end{array}$ & $\mathrm{x}$ & & MA \\
\hline Spaulding et Esposito & $\mathrm{x}$ & & $\mathrm{MA} / \mathrm{CA}$ \\
\hline Luticola doliiformis Kopalová \& Van de Vijver & $\mathrm{x}$ & & MA \\
\hline Luticola evkae Kopalová & $\mathrm{x}$ & & MA \\
\hline Luticola gigamuticopsis Van de Vijver & $\mathrm{x}$ & & MA \\
\hline
\end{tabular}


Luticola higlerii Van de Vijver, Van Dam \& Beyens $\quad$ x $\quad$ MA

Luticola katkae Van de Vijver \& Zidarova $\quad$ x

Luticola muticopsis (Van Heurck) D.G.Mann $\quad \mathrm{x} \quad \mathrm{SH}$

Luticola permuticopsis Kopalová \& Van de Vijver $\quad$ x

Luticola pusilla Van de Vijver, Kopalová, Zidarova \&

Levkov

MA

Luticola tomsui Kopalová

MA

Luticola truncata Kopalová \& Van de Vijver $\quad$ x $\quad$ MA

Luticola vermuelenii Van de Vijver $\quad \mathrm{x} \quad$ MA

Muelleria austroaltlantica Van de Vijver \& S.A.
Spaulding

Muelleria luculenta S.A.Spaulding \& J.P.Kociolek $\quad \mathrm{x} \quad$ MA

Muelleria regigeorgiensis Van de Vijver \& Spaulding $\quad \mathrm{x} \quad$ MA

Navicula gregaria Donkin $\quad \mathrm{x} \quad \mathrm{C}$

Navicula romanedwardii Zidarova, Kopalová \& Van de
Vijver

Nitzschia commutata Grunow $\quad \mathrm{x} \quad \mathrm{C}$

$\begin{array}{lll}\text { Nitzschia homburgiensis Lange-Bertalot } \quad \mathrm{x} & \mathrm{C}\end{array}$

Nitzschia paleacea Grunow $\quad \mathrm{x} \quad \mathrm{C}$

Nitzschia perminuta (Grunow) M. Peragallo x $\quad$ C

Nitzschia soratensis E. Morales \& Vis $\quad \mathrm{x} \quad \mathrm{C}$

Nitzschia vancauwenberghiana Hamsher et al. $\quad \mathrm{x} \quad$ MA

$\begin{array}{lll}\text { Orthoseira roeseana (Rabenhorst) O'Meara } & \text { x } & \text { C }\end{array}$

Pinnularia australoschoenfelderi Zidarova, Kopalová \&

Van de Vijver

$\mathrm{x}-\mathrm{MA}$

Pinnularia borealis var. pseudolanceolata B. Van de

Vijver \& R.Zidarova $\quad \mathrm{x}$

Pinnularia borealis var. scalaris (Ehrenberg) Rabenhorst $\quad \mathrm{x}$

Pinnularia subaltiplanensis Zidarova, Kopalová \& Van

de Vijver

Psammothidium rostrogermainii Van de Vijver,

Kopalová \& Zidarova $\quad \mathrm{x}$

MA

Stauroneis latistauros Van de Vijver \& Lange-Bertalot $\quad \mathrm{x} \quad$ MA/CA

Stauroneis pseudoschimanskii Van de Vijver \& Lange-

Bertalot

x $\quad$ MA

Staurosirella antarctica Van de Vijver \& E. Morales $\quad \mathrm{x} \quad$ MA

Table 2. List of all species observed in this study together with their biogeographical distribution. Distribution: CA - Antarctic Continent, MA - Maritime Antarctic Region, SH - Southern Hemisphere, C - Cosmopolitan, U - Unknown. 


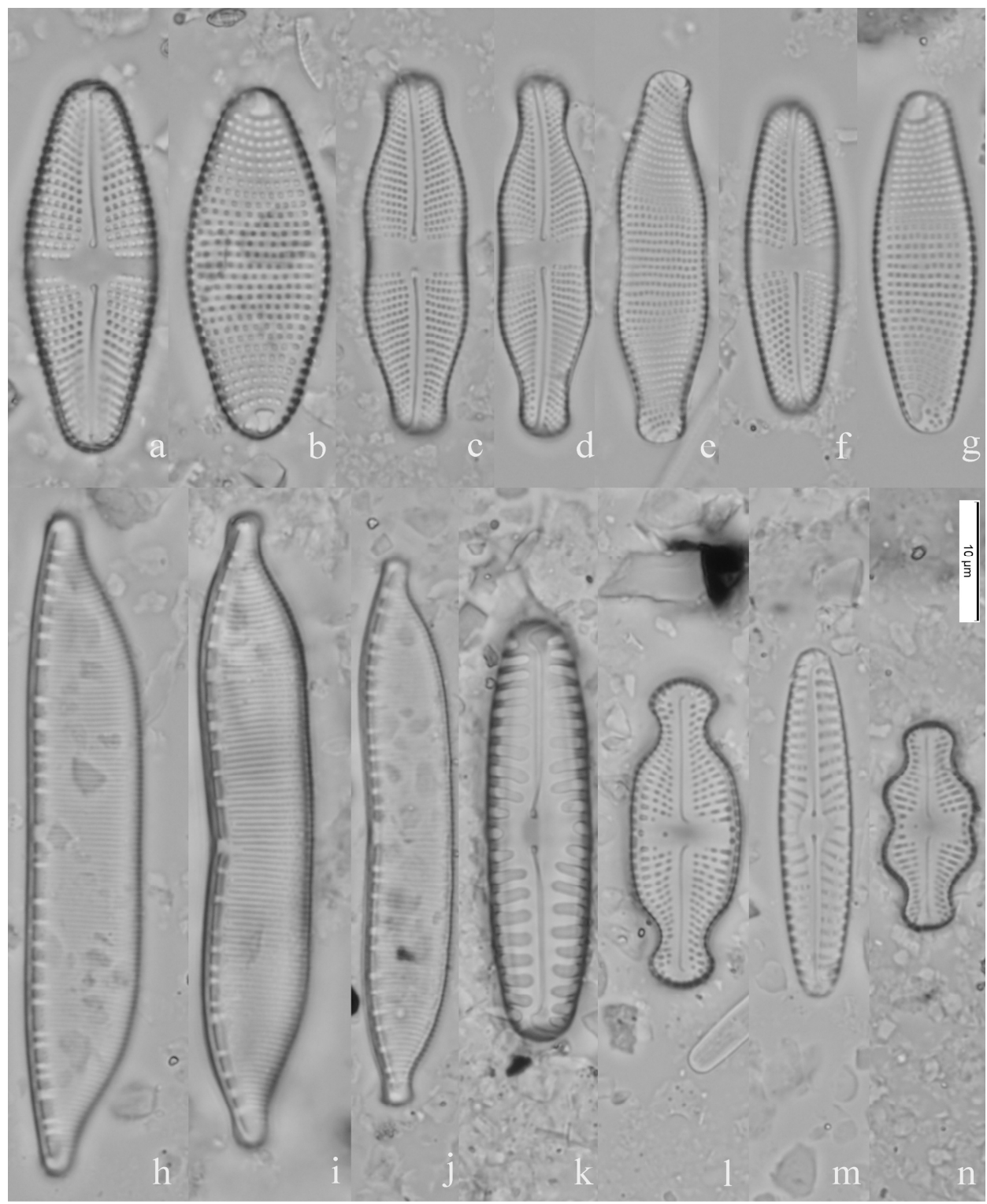

Fig. 3. a - Achnanthes muelleri raphe valve, $\mathrm{b}$ - Achnanthes muelleri rapheless valve, $\mathrm{c}, \mathrm{d}$ Achnanthes coarctata raphe valve, e - Achnanthes coarctata rapheless valve, $\mathrm{f}$ - Achnanthes taylorensis raphe valve, $\mathrm{g}$ - Achnanthes taylorensis rapheless valve, $\mathrm{h}$ - Hantzschia hyperaustralis, $\mathrm{i}$ - Hantzschia abundans, $\mathrm{j}$ - Hantzschia amphioxys f. muelleri, $\mathrm{k}$ - Pinnularia borealis, 1 - Luticola muticopsis, $\mathrm{m}$-Navicula romanedwardii, $\mathrm{n}$ - Luticola tomsui. 


\begin{tabular}{lcc}
\hline Genus & $\mathbf{n}$ & $\mathbf{\%}$ \\
\hline Luticola & 14 & 25 \\
Humidophila & 7 & 13 \\
Nitzschia & 6 & 11 \\
Hantzschia & 5 & 9 \\
Pinnularia & 4 & 7 \\
Achnanthes & 3 & 5 \\
Muelleria & 3 & 5 \\
Stauroneis & 3 & 5 \\
Halamphora & 2 & 3 \\
Navicula & 2 & 3 \\
Brachysira & 1 & 2 \\
Caloneis & 1 & 2 \\
Denticula & 1 & 2 \\
Fragilaria & 1 & 2 \\
Chamaepinnularia & 1 & 2 \\
Orthoseira & 1 & 2 \\
Psammothidium & 1 & 2 \\
\hline Total & $\mathbf{5 6}$ & $\mathbf{1 0 0}$
\end{tabular}

Table 3. Genera ordered by decreasing percental portion (\%) calculated on the number of the taxa (n).

\section{Discussion}

Based on the relative abundance data, the principal taxon was Luticola muticopsis, a cosmopolitan species, frequently reported from Antarctica and typically found in terrestrial habitats influenced by sea birds and sea sprays (Zidarova et al. 2016). The dominant taxa are typical terrestrial species reported to be frequent also in microbial mat samples on JRI (Skácelová et al. 2015) and soils, where Chattová et al. (2016) found 86 diatom taxa. Such relatively high number of typical terrestrial species of diatoms found in the lichen samples is not surprising, considering the fact that the lichen microhabitat can be characterized by extreme environmental conditions, mainly by unstable moisture regime. The diatom diversity of the James Ross Islands lichen inhabiting diatom flora is comparable with streams and seepages of
James Ross Island where Kopalová et al. (2012) found 69 taxa. When compared with diatom communities reported from lake ecosystems by Kopalová et al. (2013), samples in this study show somewhat lower species richness.

Dodd et Stoermer (1962) list in the first study focused on lichen-inhabiting diatom flora thirteen diatom species inhabiting the surface of a lichen identified as Collema sp. The samples were dominated by typical terrestrial species Achnanthes coarctata (Brébisson ex W. Smith) Grunow and Hantzschia amphioxys, similar communities characterized by the dominance of the species of genera Achnanthes and Hantzschia were reported also from James Ross Island. Lakatosh et al. (2004) identified eighteen diatom species belonging to nine genera inside the thallus of the three crus- 
tose lichens Thelotrema alboolivaceum Vain., Cryptothecia rubrocincta (Ehrenb.) Thor and Phylopsora corallina (Eschw.) Müll. Arg in neotropical lowland rain forests and discuss the potential benefits both diatoms and lichens could derive from symbiosis. They report typical cosmopolitan terrestrial taxa and six unidentified species, which can be probably new to science. Four of the typical cosmopolitan taxa are shared with the James Ross Islands lichen diatom flora- Humidophila contenta (Grunow) Lowe et al. (former Diadesmis contenta), Orthoseira roeseana (Rabenhorst) O'Meara, Pinnularia borealis and Hantzschia amphioxys. The most species rich lichen-inhabiting diatom communities (313 species) report Bertrand et al. (2016) from five lichen genera- Evernia, Usnea, Ramalina, Cladonia and Pseudevernia, collected in France. The communities were generally dominated by Pinnularia borealis and Hantzschia amphioxys, species playing an important role also on James Ross Island. However, differences can be found in the associated diatom flora of the dominant species, Bertrand et al. report Achnanthidium minutissimum (Kützing) Czarnecki, Humidophila gallica (W. Smith) Lowe et al. and Luticola goeppertiana (Bleisch) D. G. Mann ex J. Rarick et al., species absent in the lichen inhabiting flora of JRI.

The obtained results confirm the presence of a typical and highly specific nonmarine diatom flora in the Antarctic Region. For future research, the author suggests a more extensive sampling campaign, including a low temperature SEM observation and detection of diatoms within the thallus, between lichen filaments.

\section{References}

AmbrožovÁ, K., LÁsKA, K. (2016): The air temperature change on James Ross Island within the context of the Antarctic Peninsula. In: A. Nováček (ed.): Sborník príspěvků z výroční konference ČGS, 5-7 September 2016, Jihočeská Univerzita, České Budějovice, pp. 20-25. (In Czech).

BArták, M., VÁczi, P., StachoŇ, Z. and KubeŠovÁ, S. (2015): Vegetation mapping of mossdominated areas of northern part of James Ross Island (Antarctica) and a suggestion of protective measures. Czech Polar Reports 5(1): 75-87.

Bertrand, J., Coste, C., Le Cohu, R., Renon, J. P. and Ector, L. (2016): Étude préliminaire sur la présence de diatomées sur les lichens. Botany Letters, 163(2): 93-115. (In French).

Bohuslavová, O., Šmilauer, P. and Elster, J. (2012): Usnea lichen community biomass estimation on volcanic mesas, James Ross Island, Antarctica. Polar Biology, 35(10): 1563-1572.

Chattová, B., Kopalová, K. and Van de ViJver, B. (2016): Terrestrial diatom communities from Ulu Peninsula (James Ross Island, NE Antarctic Peninsula). In: Book of Abstracts SCAR Open Science Conference. Kuala Lumpur: Ministry of Science, Technology and Innovation, Malaysia, pp. 450-451. ISBN 978-0-948277-32-0.

DodD, J. D., Stoermer, E. F. (1962): Notes on Iowa diatoms I. An interesting collection from a moss-lichen habitat. Proceedings of the Iowa Academy of Science, 69: 83-87.

HrbáčeK, F., KŇAŽKovÁ, M., NÝvlt, D., LÁska, K., Mueller, C. W. and Ondruch, J. (2017): Active layer monitoring at CALM-S site near JG Mendel Station, James Ross Island, eastern Antarctic Peninsula. Science of the Total Environment, 601: 987-997.

JONES V. J. (1996): The diversity, distribution and ecology of diatoms from Antarctic inland waters. Biodiversity and Conservation, 5: 1433-1449.

Kavan, J., Ondruch, J., Nývlt, D., Hrbáček, F., CArrivick, J. L. and LÁska, K. (2017): Seasonal hydrological and suspended sediment transport dynamics in proglacial streams, James Ross Island, Antarctica. Geografiska Annaler Serie A Physical Geography, 97(1): 38-55.

Kopalová, K., Veselá, J., Elster, J., Nedbalová, L., KomáreK, J. and Van De Vijver, B. (2012): Benthic diatoms (Bacillariophyta) from seepages and streams on James Ross Island (NW Weddell Sea, Antarctica). Plant Ecology and Evolution, 145: 190-208. 
Kopalová, K., Nedbalová, L., NÝvlt, D., Elster, J. and Van De Vijver, B. (2013): Diversity, ecology and biogeography of the freshwater diatom communities from Ulu Peninsula (James Ross Island, NE Antarctic Peninsula). Polar Biology, 36: 933-948.

Kopalová, K., Ochyra, R., NedbalovÁ, L. and Van De Vijver, B. (2014): Moss-inhabiting diatoms from two contrasting Maritime Antarctic islands. Plant Ecology and Evolution, 147: 67-84.

Lakatos, M., Lange-Bertalot, H. and Büdel, B. (2004): Diatoms living inside the thallus of the green algal lichen Coenogonium linkii in neotropical lowland rain forests. Journal of phycology, 40(1): 70-73.

Rabassa, J., Skvarca, P., Bertani, L. and Mazzoni, E. (1982): Glacier inventory of James Ross and Vega Islands, Antarctic Peninsula. Annals of Glaciology, 3: 260-264.

SkÁcelová, K., HrbáČEK, F., ChATtovÁ, B., LÁsKa, K. and BARTÁK, M. (2015): Biodiversity of freshwater autotrophs in selected wet places in northern coastal ecosystems of James Ross Island. Czech Polar Reports, 5(1): 12-26.

VAN DE VIJVER, B., BeYens, L. (1999a): Biogeography and ecology of freshwater diatoms in Subantarctica: a review. Journal of Biogeography, 26: 993-1000.

Van DE ViJver, B., Beyens, L. (1999b): Moss diatom communities from Ile de la Possession (Crozet, sub-Antarctica) and their relationship with moisture. Polar Biology, 22: 232-240.

VAn DE Vijver, B., Mataloni, G., Stanish, L. and Spaulding, S. A. (2010): New and interesting species of the genus Muelleria (Bacillariophyta) from the Antarctic region and South Africa. Phycologia, 49(1): 22-41.

Van De Vijver, B., Zidarova, R. and DE HaAn, M. (2011): Four new Luticola taxa (Bacillariophyta) from the South Shetland Islands and James Ross Island (Maritime Antarctic Region). Nova Hedwigia, 92: 137-158.

VAN DE ViJver B., Zidarova R. and Kopalová K. (2014): New species in the genus Muelleria (Bacillariophyta) from the Maritime Antarctic Region. Fottea, 14: 77-90.

VAN DER WERFF (1955): A new method for cleaning and concentrating diatoms and other organisms. Verhandlungen der Internationalen Vereinigung für theoretische und angewandte Limnologie, 12: 276-277.

Zidarova, R., Van De ViJver, B., Quesada, A. and de HaAn, M. (2010): Revision of the genus Hantzschia (Bacillariophyceae) on Livingston Island (South Shetland Islands, Southern Atlantic Ocean). Plant Ecology and Evolution, 143: 318-333.

Zidarova, R., Kopalová, K. and VAN DER ViJVer, B. (2016): Diatoms from the Antarctic region: maritime Antarctica. Bibliotheca Diatomologica, 28: 1-504.

\section{Web sources / Other sources}

[1] Antarctic Digital Database, 2018. http://www.add.scar.org/ (accessed 15.11.18)

[2] Czech Geological Survey, 2009. James Ross Island - northern part. Topographic map 1: 25000.

First edition. Praha, Czech Geological Survey. ISBN 978-80-7075-734-5. 